\title{
Mycobacterium xenopi infection in an immunosuppressed patient with Crohn's disease
}

\author{
C J Majoor, A J M Schreurs, G Weers-Pothoff
}

Thorax 2004;59:631-632. doi: 10.1136/thx.2003.010546

A 48 year old patient with active Crohn's disease presented with bilateral nodules over his lungs resembling malignant metastasis. Bronchoscopic and pathological examination of the airways and sputum did not show any malignancy. After 6 weeks Mycobacterium xenopi was cultured from his bronchial washings while all other cultures remained negative. Treatment was started with rifampicin, ethambutol, and clarithromycin and, after 9 months of treatment, there was an almost complete resolution of his chest radiograph.

M ycobacterium xenopi is a water related mycobacterium, recognised as a human pathogen with low pathogenicity in 1965. It was first cultured in immunocompromised patients with lymphoma, renal transplants, or HIV infection in which a haematogenous spread of the microorganism is suggested. ${ }^{12}$ The principal risk group appears to be young severely immunocompromised individuals in whom $M$ xenopi infection probably occurs as an opportunistic infection together with other microorganisms and is even disseminated at times. It has increasingly been recognised as a cause of pulmonary infection among those with impaired immunity or chronic lung disease in whom it can colonise the airways. ${ }^{3}$

We present a case of $M$ xenopi infection in a patient with Crohn's disease resembling diffuse nodules in both lungs. To our knowledge, this radiological presentation has not previously been reported in the literature, although infection with $M$ xenopi is not exceptionally rare.

\section{CASE REPORT}

A 48 year old man was referred to our outpatient clinic with abnormalities on his chest radiograph. He complained of expectorations of green sputum, an afternoon rise in temperature, and a sore throat which did not improve over a course of antibiotics. Besides slight tiredness and minor dyspnoea, he denied all other complaints.

His medical history included Crohn's disease with several exacerbations in previous years for which he had undergone surgical resection of the ileocaecal and neoterminal ileum. He was receiving treatment with azathioprine and prednisone, but no other medications. He had no known allergies, had no pets, and was the owner of a steel construction company.

Physical examination showed a healthy man with no dyspnoea who was slightly pale but had normal blood pressure, pulse and temperature. Other than some ronchi over his lungs which cleared up after coughing, no abnormalities were found.

Laboratory findings at presentation showed a haemoglobin level of $8.6 \mathrm{mmol} / \mathrm{l}$, leucocytes $9.0 \times 10^{9} / \mathrm{l}$, and normal kidney function, electrolytes and liver enzymes, although an increased erythrocyte sedimentation rate $(59 \mathrm{~mm} / \mathrm{min})$, progressive normocytic anaemia (haemoglobin $7.3 \mathrm{mmol} / \mathrm{l}$ ), and a slight increase in his liver enzymes developed later. An arterial blood gas sample was normal.

The chest radiograph (fig l) showed diffuse nodular consolidations in both lungs, and a computed tomographic (CT) scan (fig 2) confirmed multiple nodular lesions of approximately $1 \mathrm{~cm}$ diameter. Bronchoscopic examination and bronchoalveolar lavage (BAL) showed no endobronchial aberrations and pathological examination of the BAL fluid found no abnormalities. A percutaneous needle biopsy showed no malignancy and an open lung biopsy was suggested several times but refused by the patient.

The sputum and BAL fluid were cultured for bacterial (including Actinomyces and Nocardia), fungal, and mycobacterial microorganisms. The only positive culture was a mycobacterium after 6 weeks of incubation. Polymerase chain reaction for Mycobacterium tuberculosis and ZiehlNielsen staining of the bronchial washings and sputum samples were negative and a PPD skin test was also negative at presentation. Serological examination for Aspergillus and viruses was negative.

Treatment was started with isoniazid $300 \mathrm{mg}$, rifampicin $600 \mathrm{mg}$, ethambutol $1600 \mathrm{mg}$, pyrazinamide $2000 \mathrm{mg}$, and pyridoxine $20 \mathrm{mg}$. After the mycobacterium was identified as $M$ xenopi and the in vitro drug susceptibility of the strain was assessed, the regimen was changed to rifampicin, ethambutol, and clarithromycin $1000 \mathrm{mg}$. After 9 months there was almost complete resolution of his chest radiograph so treatment was stopped. He is followed up closely as relapse has been described after long periods of time.

\section{DISCUSSION}

Mycobacterium xenopi infections can present in two wayseither with cough, malaise, weight loss and haemoptysis over a period of months, or with chronic dyspnoea, weight loss and chronic cough with chest radiographic abnormalities over a period of years. ${ }^{245}$ Radiographic abnormalities in nontuberculosis mycobacterial infections are numerous and include mediastinal or hilar adenopathy, heterogeneous and linear pulmonary areas of increased opacity, cavitation, and miliary nodules. ${ }^{346}$ Costrini et $^{4} \mathrm{l}^{7}$ even found (multi) nodular masses resembling neoplasm, although these lesions were situated in the upper lobes and were not diffusely affecting the lungs as in our patient.

The appearance of the CT scan suggested massive pulmonary metastasis, although there were no further clues in the laboratory tests or physical examination. The clinical and radiological features were compatible with granulomatous disease in an immunosuppressed patient (sarcoidosis) but no clues were identified in the fine needle biopsy specimen or BAL fluid. An open lung biopsy was refused by the patient at first and later postponed, becoming unnecessary when the cultures were positive and the chest radiograph improved on treatment. Pulmonary lesions in inflammatory bowel disease have been described but usually have a different pulmonary presentation such as interstitial 


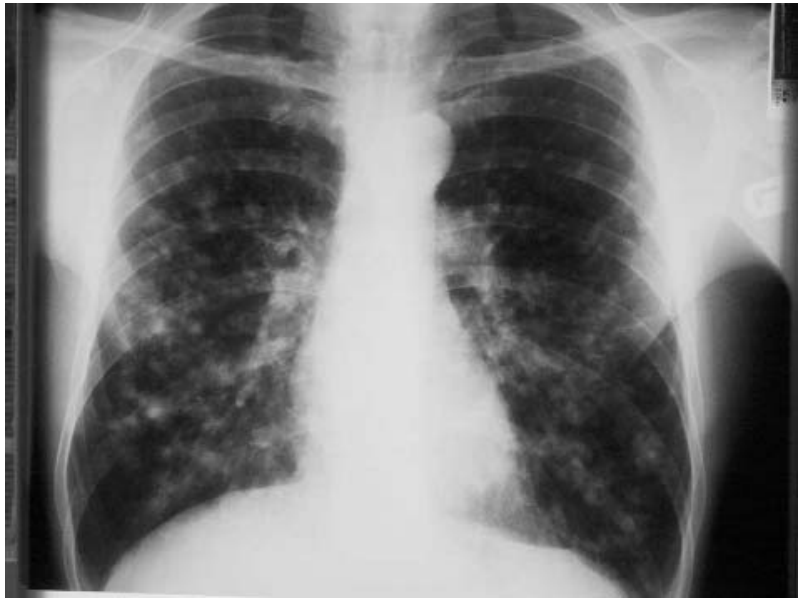

Figure 1 Chest radiograph showing diffuse nodular consolidations in both lungs.

disease, bronchiectasis, bronchiolitis obliterans organising pneumonia (BOOP), pulmonary oedema, alveolar consolidation, or granulomatous interstitial involvement. ${ }^{8}$ The appearance of multiple lung masses in Crohn's disease has been described but is very uncommon. ${ }^{9}$ Other infections to consider besides the more common infectious agents are Nocardia, Aspergillus fumigatus, other fungi (Coccidiomycosis, Cryptococcosis and Histoplasmosis), and measles, but cultures and serological examinations for these were negative.

Diagnosis is made by culture from sputum or BAL fluid in the absence of other pathogenic microorganisms. Treatment should be started with a multiple drug regimen based on in vitro drug susceptibility of the strain, remembering that the in vivo response to the drug regimen is not always related to the in vitro results. Treatment should be given for at least 9 months, but no definitive period has been assessed. ${ }^{10}$ The prognosis of $M$ xenopi is uncertain. Relapse frequently occurs, but this has not been studied in a large series since $1984 .^{2}$

\section{Authors' affiliations}

C J Majoor, A J M Schreurs, G Weers-Pothoff, Department of Pulmonary Diseases, Jeroen Bosch Hospital, 's Hertogenbosch, The Netherlands

Correspondence to: Dr C J Majoor, Department of Pulmonary Diseases, Jeroen Bosch Hospital, Postbus 90153, 's Hertogenbosch 5200 ME, The Netherlands; cjmajoor@planet.nl

Received 6 June 2003

Accepted 14 August 2003

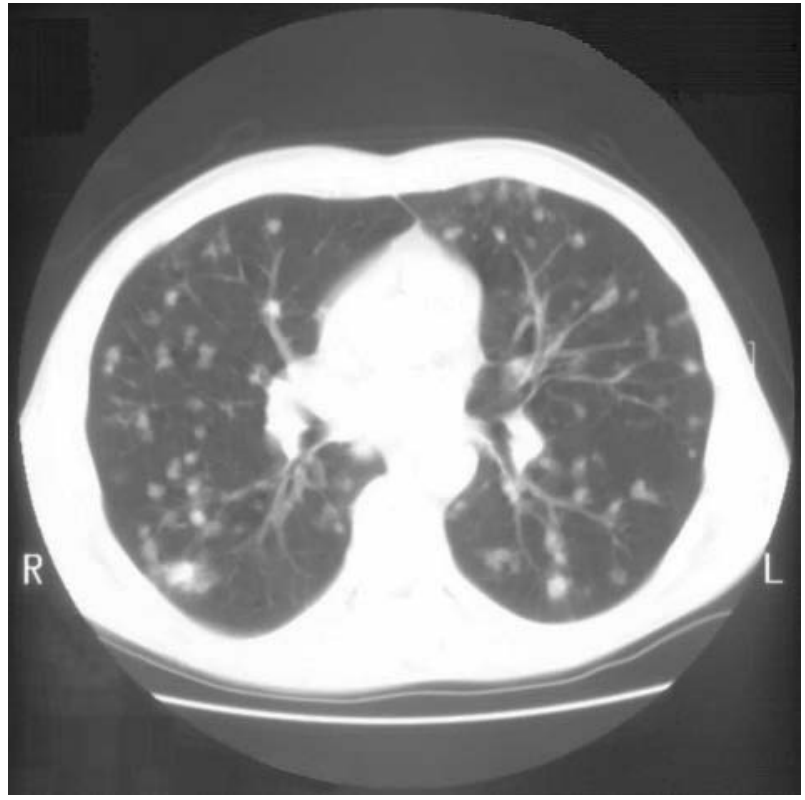

Figure 2 CT scan of the thorax showing multiple nodular lesions.

\section{REFERENCES}

1 Damsker B, Bottone EJ, Deligdisch L. Mycobacterium xenopi: infection in an immunocompromised host. Hum Pathol 1982;13:866-70.

2 Banks J, Hunter AM, Campbell IA, et al. Pulmonary infection with Mycobacterium xenopi: review of treatment and response. Thorax 1984;39:376-82.

3 Jiva TM, Jacoby HM, Weymouth LA, et al. Mycobacterium xenopi: innocent bystander or emerging pathogen? Clin Infect Dis 1997;24:226-32.

4 Contrevas MA, Cheung OT, Sanders DE, et al. Pulmonary infection with nontuberculous mycobacteria. Am Rev Respir Dis 1988;137:149-52.

5 Smith J, Citron KM. Clinical review of pulmonary disease caused by Mycobacterium xenopi. Thorax 1983;38:373-7.

6 Erasmus JJ, McAdams HP, Farrell MA, et al. Pulmonary nontuberculous mycobacterium infection: radiologic manifestations. Radiographics 1999:19:1487-505.

7 Costrini AM, Mahler DA, Gross WM, et al. Clinical and roentgenographic features of nosocomial pulmonary disease due to Mycobacterium xenopi. Am Rev Respir Dis 1981;213:104-9.

8 Mahadeva R, Walsh G, Flower CDR, et al. Clinical and radiological characteristics of lung disease in inflammatory bowel disease. Eur Respir J 2000;15:41-8

9 Lucero PF, Frey WC, Shaffer RT, et al. Granulomatous lung masses in an elderly patient with inactive Crohn's disease. Inflamm Bowel Dis 2001;7:256-9.

10 Research Committee of the British Thoracic Society. First randomised trial of treatment for pulmonary disease caused by $M$ avium intracellulare, $M$ malmoense, and $M$ xenopi in HIV negative patients: rifampicin, ethambutol and isoniazid versus rifampicin and ethambutol. Thorax 2001;56:167-72. 